\title{
Model colour-magnitude diagrams of elliptical galaxies ${ }^{\star}$
}

\author{
C. Ikuta
}

\author{
National Astronomical Observatory of Japan (NAOJ), 2-21-1 Osawa, Mitaka, Tokyo 181-8588, Japan \\ e-mail: chisato.ikuta@nao.ac.jp
}

Received 30 December 2005 / Accepted 15 May 2007

\begin{abstract}
Context. Formation and evolution of elliptical galaxies are still matters of debate. Knowledge of their star formation histories (SFHs) is essential to understand the formation mechanism. Colour-magnitude (C-M) diagrams of elliptical galaxies should provide one of the most important clues to resolve the SFHs of the elliptical galaxies.

Aims. The aim of this study is to illustrate what information can be obtained from C-M diagrams of elliptical galaxies and what differences appear in theoretical C-M diagrams of different formation scenarios.

Methods. Using a C-M diagram simulator which consistently solves SFH and chemical evolution, we present and discuss six examples of model $\mathrm{C}-\mathrm{M}$ diagrams. The $\mathrm{C}-\mathrm{M}$ diagrams can be considered a first approach to the theoretical C-M diagrams of elliptical galaxies of which formation is described as the monolithic collapse and the hierarchical scenarios.

Results. We show that there are three main differences in the C-M diagrams between the two formation scenarios; the magnitude of main sequence turnoff (MSTO), horizontal branch (HB) morphology, and colour distribution of red giant branch (RGB) stars. Since a later epoch of the final star formation is assumed in the hierarchical model, the magnitude of the MSTO is brighter than that of the monolithic collapse. The C-M diagram predicted by the hierarchical scenario shows well-defined blue HB. On the other hand, the C-M diagram shows few blue HB stars in the monolithic collapse case. The colour distributions predicted by the former have bluer peaks than those by the latter. We find that this corresponds to a higher frequency of metal-poor stars in the former model and metal-poor populations are born in the progenitor galaxies. In addition to the model C-M diagrams, we discuss metallicity distributions and $\alpha / \mathrm{Fe}$ abundance patterns predicted by the two scenarios.
\end{abstract}

Key words. galaxies: formation - galaxies: evolution - galaxies: elliptical and lenticular, cD stars: Hertzsprung-Russell (HR) and C-M diagrams - galaxies: stellar content

\section{Introduction}

Formation and evolution of giant elliptical galaxies are still matters of debate. Historically, there are two views on elliptical formation; the monolithic (e.g., Tinsley 1972; Larson 1974) and the hierarchical (e.g., Toomre 1977; Kauffmann et al. 1993) scenarios. The modern version of the classical "monolithic collapse scenario" puts the stress on an elliptical assembly from gaseous material (i.e., with dissipation), in the form of either a unique cloud or many gaseous clumps, but not out of preexisting stars. The competing "hierarchical scenario" proposes that galaxies form hierarchically through successive non-dissipative, random mergers of subunits over a wide redshift range in such a way that more massive ones (i.e., ellipticals) are more likely to form at later through mergers of preexisting disc galaxies.

The SFH provides essential information for understanding the evolution of elliptical galaxies. The age distribution of stellar populations in ellipticals is a key piece in the puzzle of the origins and evolution of ellipticals. The most reliable way to derive SFHs of galaxies is from a study of the resolved stellar content. Ideally, this would be the first step in correctly interpreting their integrated light by means of spectral synthesis techniques which is generally used to analyse observations of ellipticals at large distances (e.g., Trager et al. 2000; Kuntschner et al. 2002; Dressler et al. 2004).

\footnotetext{
* Appendix A is only available in electronic form at http: //www . aanda.org
}

Simulation using C-M diagrams was pioneered by MeyerHofmeister (1969) and was first applied by Tosi et al. (1991) for analysis of stellar populations in a Local Group dwarf galaxy. This has become the standard technique to study SFHs of galaxies in the Local Group. Experiments of modelling C-M diagrams of more complex systems have been also presented. For example, Hernandez et al. (2000) and Bertelli \& Nasi (2001) reported the SFH of the solar vicinity, i.e., the Galactic disc, by analysing Hipparcos C-M diagrams of field stars in the solar vicinity. The SFH of the Galactic bulge was studied using a similar technique (Bertelli et al. 1995; $\mathrm{Ng}$ et al. 1996).

SFHs of various systems from smaller to larger more complex ones have been studied based on C-M diagrams except for massive and early-type galaxies, i.e., elliptical and S0 galaxies. Even with the best available imaging tools such as the Hubble Space Telescope, resolving stars is only possible for less crowded halo populations in the nearest E/S0 galaxies (e.g., Soria et al. 1996; Davidge \& van den Bergh 2001; Gregg et al. 2004; Schulte-Ladbeck et al. 2003; Harris \& Harris 1999, 2000, 2002), while integrated light measurements refer to the central areas. Although it is currently very challenging, next generation telescopes and/or instruments will enable us to study an elliptical galaxy individual-star-basis from the central region to the outer.

Here, we explore differences in C-M diagrams of elliptical galaxies assuming the monolithic and hierarchical formation scenarios. The aim of this study is to determine properties of $\mathrm{C}-\mathrm{M}$ diagrams of ellipticals of different formation in prior to future observations. Based on model C-M diagrams, we show 
three main differences; colour distribution of RGB stars, HB morphology, and magnitude of MSTO. Colour distribution predicted by the monolithic collapse model has a redder peak than that predicted by the hierarchical model due to a larger fraction of metal-rich stars. Star formation and chemical enrichment histories affect the HB morphology. The monolithic collapse model predicts fewer blue horizontal branch stars, while the hierarchical expects populated blue horizontal branch stars as well as red ones. Since we assume later formation epoch of ellipticals in the former model, the magnitude of the main sequence turn-off appears brighter than that of the latter.

Calculated metallicity distributions also differ. The monolithic collapse scenario predicts a metallicity distribution of a single peak and a small fraction of metal-poor $([\mathrm{Z} / \mathrm{H}]<-1.0)$ stars. On the other hand, the hierarchical scenario predicts distribution with two peaks. The first peak at lower metallicity corresponds to the yield of progenitor galaxies, while the second one corresponds to a starburst when the progenitor galaxies collide and merge. Both scenarios predict super-solar $\alpha / \mathrm{Fe}$ ratio, and the monolithic collapse models predict higher values of $\alpha / \mathrm{Fe}$. The averaged values of all long-lived stars are $[\mathrm{Mg} / \mathrm{Fe}]=0.54-0.56$ in the monolithic collapse models, while $[\mathrm{Mg} / \mathrm{Fe}]=0.30-0.42$ in the hierarchical models.

Throughout this paper, we use the following cosmological parameters unless otherwise stated; $H_{0}=71 \mathrm{~km} \mathrm{~s}^{-1} / \mathrm{Mpc}, \Omega_{0}=$ $1.0, \Omega_{\mathrm{m}}=0.27$.

\section{Simulator of a colour-magnitude diagram}

Our simulator of C-M diagrams fully takes into account the effects of chemical evolution and consistently calculates an assumed SFH and chemical evolution. Stars form, chemical enrichment proceeds as massive stars explode as supernovae, and new generations of stars form from metal enriched gas. Stars change their spectral properties as they evolve, according to their initial mass and metallicity. The distribution of stars in the Hertzsprung-Russell diagram at a given time is calculated from stellar evolutionary tracks. The magnitudes of the stars are calculated in a series of filters using grids of stellar model atmospheres referring to the appropriate metallicity, effective temperature, and surface gravity. Therefore, the distribution of stars in the C-M diagram (i.e., the Hess diagram) can be obtained at any time.

The structure of our C-M diagram simulator is briefly given below. More details of the chemical evolution code and of the $\mathrm{C}$ M diagram simulator are described in Ikuta \& Arimoto (2000) and Ikuta \& Arimoto (2002), respectively. (1) A SFH and chemical enrichment history is calculated by assuming a star formation law. Nucleosynthesis data is taken from Thielemann et al. (1996) for type II supernovae, and from Nomoto et al. (1984) for type Ia supernovae. No instantaneous recycling is assumed. The delayed enrichment by type Ia supernovae is taken into account. Here we assume $1.5 \mathrm{Gyr}$ as the life time of progenitors of type Ia supernovae which reproduce $\alpha / \mathrm{Fe}$ versus iron abundance relation observed in the Galactic halo and disc stars. (2) A HR diagram is simulated and a Monte Carlo simulation is performed to assign stellar masses. The stellar evolutionary tracks by the Padova group (Bressan et al. 1993; Fagotto et al. 1994a,b; Girardi et al. 1996) are used for metallicity less than $z=0.02$, while those of the Yale group (Yi et al. 2001) are adopted for higher metallicity. The original isochrones are interpolated in metallicity in order to cover the entire range needed of ages and metallicities. The later stages of stellar evolution, in particular, $\mathrm{HB}$, asymptotic giant branch (AGB), and post-AGB stars are treated with care. The HB
Table 1. Photometric errors and detection completeness input into the simulation code of colour-magnitude diagrams. (1) absolute magnitude; (2) photometric errors in $V$; (3) detection completeness in $V$; (4) photometric error in $I$; (5) detection completeness in $I$.

\begin{tabular}{ccccc}
\hline \hline $\begin{array}{c}\text { Mag } \\
(1)\end{array}$ & $\begin{array}{c}V \\
(2)\end{array}$ & $\begin{array}{c}\text { V.C. } \\
(3)\end{array}$ & $\begin{array}{c}I \\
(4)\end{array}$ & $\begin{array}{c}I . C . \\
(5)\end{array}$ \\
\hline-3. & 0.015 & 0.98 & 0.015 & 0.99 \\
-2. & 0.027 & 0.99 & 0.027 & 0.99 \\
-1. & 0.030 & 0.98 & 0.030 & 0.98 \\
0. & 0.030 & 0.98 & 0.030 & 0.98 \\
1. & 0.030 & 0.98 & 0.030 & 0.90 \\
2. & 0.030 & 0.98 & 0.050 & 0.80 \\
3. & 0.050 & 0.88 & 0.050 & 0.80 \\
4. & 0.050 & 0.80 & 0.050 & 0.75 \\
5. & 0.070 & 0.70 & 0.070 & 0.70 \\
6. & 0.200 & 0.50 & 0.200 & 0.50 \\
\hline
\end{tabular}

stars are distributed on the HR diagram accordingly to a modified Gaussian mass distribution equation (Lee et al. 1990) which represents the stellar distribution along the HB of Galactic globular clusters. This is equivalent to considering a statistical dispersion of mass loss rate along the RGB. Once a star leaves from a zero age horizontal branch (ZAHB), the later stellar evolution follows the track corresponding to the stellar mass on the ZAHB. There is mass distribution on the ZAHB even for stars born simultaneously with the same mass and metallicity. Therefore, a single line does not represent the stellar distribution on a HR diagram including old stellar populations. We calculate the tracks taking this into account. (3) The HR diagram is converted into the colour and magnitude plane using stellar model atmospheres taken from Lejeune et al. (1998). (4) To obtain the final C-M diagram, another Monte Carlo simulation is performed to include the photometric errors and possible incompleteness in the observations.

Table 1 shows artificial errors and detection completeness input into simulations of colour-magnitude diagrams. Note that careful simulation of crowding effects is essential to correctly interpret observed C-M diagrams. Stellar crowding is expected to play the main role imposing a limit to the photometry and affecting its quality, especially near the limiting magnitude. Crowding effects are of three kinds; detection incompleteness of stars, large errors in magnitude, and systematic shift in magnitude. These three effects are strong, nontrivial functions of the magnitude of each star. In particular, the later effect is expected to be serious for very crowded central regions of an elliptical galaxy. Such errors should be estimated from observational data, for example by running artificial star tests.

\section{Elliptical formation scenarios}

\subsection{Monolithic collapse model}

The monolithic formation picture of elliptical galaxies assumes that initial bursts of star formation occurred during the rapidly infalling stage of proto-galactic gas clouds and that the gas was expelled when the cumulative thermal energy of the remaining gas exceeded the binding energy of a galaxy (Larson 1974; Arimoto \& Yoshii 1987). No stars formed afterwards and the galaxy has evolved passively since.

As a representative of the monolithic collapse models, "metallicity sequence model" by Kodama \& Arimoto (1997, hereafter KA97) is adopted in our simulation. The metallicity sequence model assumes that metallicity difference between elliptical galaxies results in observed C-M relation, i.e., giant 
Table 2. Parameters of monolithic collapse models. (1) model ID; (2) formation epoch; (3) timescale of star formation in Gyr; (4) timescale of gas infall in Gyr; (5) epoch of galactic wind; (6) IMF slope. Parameters are taken from the metallicity sequence model by Kodama \& Arimoto (1997) except for the formation epoch.

\begin{tabular}{cccccc}
\hline \hline $\begin{array}{c}\text { model } \\
(1)\end{array}$ & $\begin{array}{c}z_{\mathrm{f}} \\
(2)\end{array}$ & $\begin{array}{c}\tau_{\mathrm{i}} \\
(3)\end{array}$ & $\begin{array}{c}\tau^{*} \\
(4)\end{array}$ & $\begin{array}{c}z_{\mathrm{gw}} \\
(5)\end{array}$ & $\begin{array}{c}x \\
(6)\end{array}$ \\
\hline $\mathrm{MC} 01$ & 8 & 0.1 & 0.1 & 6.8 & 1.10 \\
$\mathrm{MC} 02$ & 8 & 0.1 & 0.1 & 6.3 & 1.10 \\
$\mathrm{MC} 03$ & 8 & 0.1 & 0.1 & 5.1 & 1.10 \\
\hline
\end{tabular}

galaxies have higher metallicity so they are redder than fainter ones. Parameters to describe a SFH are formation epoch $T_{\mathrm{f}}$, timescale of gas infall $\tau_{\mathrm{i}}$, timescale of star formation $\tau_{*}$, epoch of the termination of star formation $T_{\mathrm{gw}}$, and the initial mass function (IMF) $\phi(m)$, which is defined by mass fraction and assumed to have a single power law of mass, $\phi(m) \mathrm{d} m \propto m^{-x} \mathrm{~d} m\left(m_{1} \leq m \leq\right.$ $m_{\mathrm{u}}$ ), where $m_{1}$ and $m_{\mathrm{u}}$ are lower and upper mass limits of initial stellar mass, respectively, and $m_{1}=0.1 M_{\odot}$ and $m_{\mathrm{u}}=60 M_{\odot}$ are assumed. The Salpeter mass function has a slope $x=1.35$ in this definition. See KA97 for more details. The so-called infall model of chemical evolution was assumed, but Ti falls short of $0.1 \mathrm{Gyr}$. The model parameters are adjusted to reproduce $V-K$ to- $V$ C-M relation observed in Coma cluster.

Table 2 shows the model parameters. Instead of the formation epoch of $15 \mathrm{Gyr}$ ago originally assumed in KA97, $13 \mathrm{Gyr}$ ago is adopted to account for the recent estimate of the universe age.

Figure 1 shows the star formation and chemical enrichment histories of model MC01-MC03 together with those of the merger models which are described in the next subsection. The $x$-axis denotes the galactic age. In the upper and lower panels, the $y$-axis gives star formation rate (SFR) and iron abundance, respectively. Since all the parameters of SFHs except for $T_{\mathrm{gw}}$ are the same, the paths of the chemical evolution are identical for the three models and only the final metallicity is different. The arrows in the lower panels indicate the final iron abundances of models MC01-MC03.

\subsection{Hierarchical model}

In the merger scenario, the primary route by which bright elliptical galaxies form is through galaxy mergers. The outcome of each merger depends on the ratio of the mass of the merging satellite, $M_{\text {sat }}$, to that of the central galaxy, $M_{\text {cet }}$, where $M_{\text {sat }}$ and $M_{\text {cet }}$ are defined in terms of stars and cold gas only. Numerical simulations (Walker et al. 1996; Barners 1998) showed that a single elliptical galaxy was produced when the mass ratio of merging galaxies was in the range of $0.3 \leq M_{\text {sat }} / M_{\text {cet }} \leq 1.0$. To simplify, we consider a specific case of $f_{\text {ellip }}=1.0$ and assume that progenitors are characterised by SFH of the Milky Way type disc.

The SFH of the progenitors are described using the following parameters; epoch starting star formation $T_{\mathrm{f}}$, timescale of gas infall $\tau_{\mathrm{i}}$, timescale of star formation $\tau_{*}$, epoch of merging $T_{\mathrm{m}}$, and the IMF slope $x$. We consider the infall model and adopt $\tau_{\mathrm{i}}=\tau *=5 \mathrm{Gyr}$ and $x=1.35$, which reproduce abundance ratios and metallicity distribution observed in the solar neighbourhood (Arimoto et al. 1992). The infall law suggested by a hierarchical model (Sommer-Larsen et al. 2003) was in good agreement with that used in chemical evolution models for the Milky Way which can reproduce the majority of the

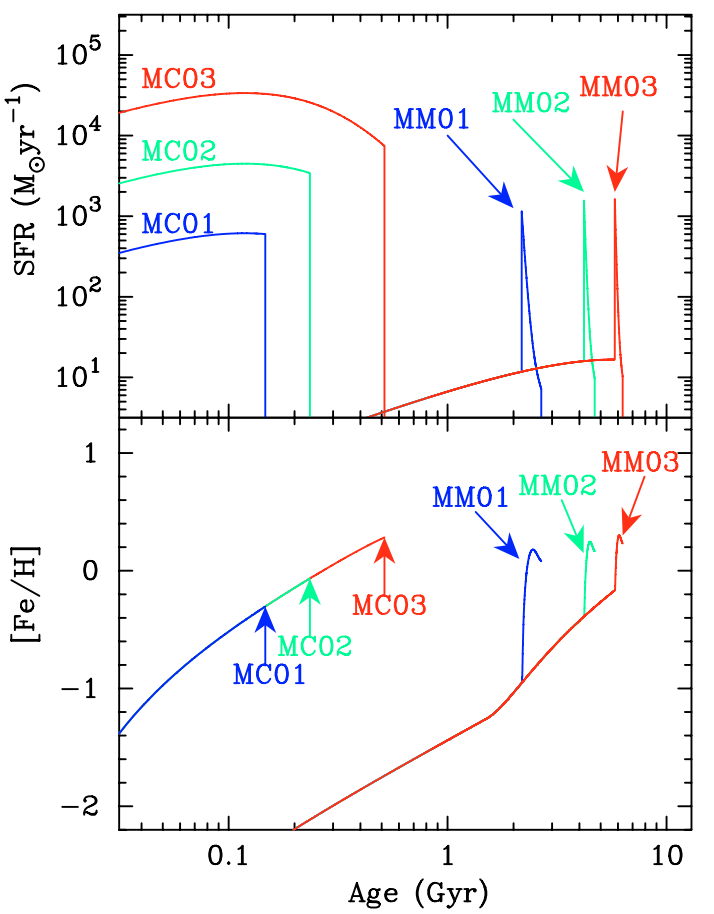

Fig. 1. Star formation (upper panel) and chemical enrichment (lower panel) histories of models MC01-MC03. (This figure is available in color in electronic form.)

observational constraints. Therefore, the approximation of the infall model adopted here is adequate for our purpose.

We focus on ellipticals at $z=0.6,0.9$, and 1.5 reported in the study by Kauffmann (1996), who concluded that the C-M relations and red-envelope populations are expected in the hierarchical simulations. In Fig. 7 of Kauffmann (1996), we identify galaxies which have been formed by major merger in the past 1.5 Gyr. From these galaxies, a galaxy on the red-envelope is picked up at each redshift. Such galaxies should be old enough to be red-envelope populations. Therefore, the merging epochs are assumed to be $1.5 \mathrm{Gyr}$ before these redshifts.

The star formation during the merger occurs in a very short timescale similar to the dynamical timescale of the elliptical that is formed (Cole et al. 2000). The dynamical timescale is estimated based on a study of present-day ellipticals. Saito (1970) analysed line-of-sight velocity dispersion and luminosity profile of spheroidal systems including ellipticals and obtained the relation of binding energy $\Omega_{\mathrm{G}}$ versus mass $M_{\mathrm{G}}$ as follows;

$\Omega_{\mathrm{G}}=1.66 \times 10^{60}\left(M_{\mathrm{G}} / 10^{12} M_{\odot}\right)^{\eta} \operatorname{erg} \quad(\eta=1.45)$.

Assuming that a formed galaxy is relaxed and become homogeneous and in dynamical equilibrium, we derive the averaged quantities of the radius $R_{\mathrm{G}}$, density $\rho$, and dynamical time $t_{\mathrm{d}}$, respectively as follows;

$R_{\mathrm{G}}=G M_{\mathrm{G}} /\left(2 \Omega_{\mathrm{G}}\right)=26.1\left(M_{\mathrm{G}} / 10^{12} M_{\odot}\right)^{2-\eta} \mathrm{kpc}$

$\rho=3 M_{\mathrm{G}} /\left(4 \Omega_{\mathrm{G}}\right)=9.15 \times 10^{-25}\left(M_{\mathrm{G}} / 10^{12} M_{\odot}\right)^{3 \eta-5} \mathrm{~g} \mathrm{~cm}^{-3}$,

$t_{\mathrm{d}}=(3 \pi / 32 G \rho)^{0.5}=6.97 \times 10^{7}\left(M_{\mathrm{G}} / 10^{12} M_{\odot}\right)^{(5-3 \eta) / 2} \mathrm{yr}$.

The dynamical time depends weakly on the galactic mass and is evaluated as $t_{\mathrm{d}}=1.6 \times 10^{7}-7.0 \times 10^{7} \mathrm{yr}$ for galaxies of $M_{\mathrm{G}}=10^{10}-10^{12} M_{\odot}$. Therefore, the timescale of star formation 
Table 3. Parameters of merger models. (1) model ID; (2) epoch to start collapsing; (3) timescale of gas infall in Gyr (4) timescale of star formation in Gyr; (5) epoch of merger event; (6) timescale of star formation after the merger in Gyr; (7) duration of star formation after the merger in Gyr; (8) IMF slope.

\begin{tabular}{cccccccc}
\hline \hline model & $z_{\mathrm{f}}$ & $\tau_{\mathrm{i}}$ & $\tau *$ & $z_{\mathrm{m}}$ & $\tau *^{\prime}$ & $\Delta T$ & $x$ \\
$(1)$ & $(2)$ & $(3)$ & $(4)$ & $(5)$ & $(6)$ & $(7)$ & $(8)$ \\
\hline MM01 & 8 & 5 & 5 & 2.35 & 0.1 & 0.1 & 1.35 \\
MM02 & 8 & 5 & 5 & 1.33 & 0.1 & 0.1 & 1.35 \\
MM03 & 8 & 5 & 5 & 0.88 & 0.1 & 0.1 & 1.35 \\
\hline
\end{tabular}

$\tau *$ is assumed to have abruptly changed to $5 \times 10^{7}$ yrs after the merger. The IMF is assumed to be the same before and after the starburst, since anomalous IMF slopes and mass cut-offs are still controversial (Brandle \& Andersen 2004; Elmegreen \& Scalo 2006). Since a negligible amount of gas remains $0.1 \mathrm{Gyr}$ after the starburst, we stop star formation $0.1 \mathrm{Gyr}$ after the merger and assume that the formed system evolves passively.

Table 3 summarises the adopted parameters, which are input into the chemical evolution code. The resulting SFHs include both quiescent star formation in the progenitors as well as the starburst triggered by the merger. Note that the chemical evolution models assume that galaxies evolve as single-body systems and this is not the case in the hierarchical models, where supernova feedback effects are thought to be important in driving chemical evolution. Feedback effects could either drive the derived $\alpha / \mathrm{Fe}$ ratios up or down, depending on the relative efficiencies with which the products of type II and type Ia supernovae are expelled.

Figure 1 shows the star formation and chemical enrichment histories of models MM01-MM03. When the progenitors merge, very high $\left(\gtrsim 10^{3} M_{\odot} \mathrm{yr}^{-1}\right)$ SFRs are expected. Such high SFRs are estimated in bright sub-millimetre galaxies if the observed sub-millimetre fluxes originated mainly from star formation (e.g., Smail et al. 2002; Chapman et al. 2003). The SFRs are comparable to those of objects observed in sub-millimetre for a region around an X-ray selected QSO at $z=1.8$ (Stevens et al. 2004). The authors suggested that galaxy mergers or encounters causes the high SFRs of these objects. Analysing 23 submillimetre galaxies and three $I S O$-detected extremely red objects using an evolutionary SED model of dusty star-bursts, Takagi et al. (2004) found that SFRs of over $10^{3} M_{\odot} \mathrm{yr}^{-1}$ are estimated in more than $60 \%$ of the sample. These results indicate that the SFRs of our models are consistent with those of a part of dusty starbursting objects.

\section{Results}

\subsection{Colour-magnitude diagrams}

Figure 2 displays stellar density on C-M diagrams. A total of 1000000 stars are generated before including the observational effects (magnitude errors and detection completeness). The upper and lower panels show the C-M diagrams of the monolithic collapse and the merger models, respectively. In the lower panels, the isochrones are included to guide the eye and mark the first populations of the starburst.

Figure 3 shows simple dotted C-M diagrams which are less populated than those in Fig. 2. In total, $90 \%$ of stars are randomly subtracted from the original calculations. In Fig. 4, some key regions in the $\mathrm{C}-\mathrm{M}$ diagrams are labelled. The fraction of stars in each box is reported in Table A.1. Tables A.2-A.7 summarise the fraction of stars of different metallicity over the total in a given box.

A clear difference between the monolithic and the merger models appears in the BHB box. In the upper panels, there is no trace of an extended blue HB, which would normally be found if an old metal-poor stellar population was present. A very small fraction of metal-poor stars results in lack of blue $\mathrm{HB}$ in the monolithic collapse model. As Fig. 1 shows, the chemical enrichment progresses rapidly, due to high initial SFR in the model. Consequently, the majority of the stellar populations have metallicity that is too high to be blue HB stars (see Fig. 6 and Table A.2, for theoretical metallicity distributions and fraction of metallicity in the BHB box, respectively). On the other hand, a well-defined blue HB stars appear in the lower panels. In the merger models, the blue HB stars originate from the population born in the progenitor galaxies. Because the metallicity gradually increases, the progenitors contain many old and metalpoor populations. This trend suggests that the HB morphology would give us a clue to distinguish the two formation scenarios of ellipticals.

Stellar concentration at the middle of the RGB appears in the C-M diagrams of model MM01-MM03. The isochrones corresponding to the population are drawn in Fig. 2 to guide the eye. They are the first populations formed during the starburst triggered by the merger. During the first few million years of the starburst, supernovae of the burst populations not yet exploded and stars form from the gas of the final abundance of the progenitors. Such stars have the same age and metallicity. This results in the stellar concentration in the C-M diagrams. After the supernovae start to explode, chemical enrichment progresses rapidly and the formed stars have similar ages but different metallicities. This makes stars of the later populations redder. We note that this is rare. In each of the models MM01-MM03, the same SFH of the progenitors is assumed, so both progenitors have the same metallicity just before the merger. It is more realistic that progenitors with different SFH and CEH merge to form an elliptical. In this case, a fewer or no stellar concentration should appear in the C-M diagram of the formed elliptical.

Due to the later termination epoch of star formation assumed in the merger models, the MSTOs $\left(3<M_{V}<3.7\right)$ of the merger models MM01-MM03 are brighter than those $\left(M_{V} \sim 4\right)$ of the monolithic collapse models MC01-MC03. The faintest and brightest absolute magnitudes of MSTOs provide the most reliable information of the epoch when star formation started and ended. Therefore, it is ideal to obtain a C-M diagram deep enough to detect at least the MSTO of the youngest populations in an elliptical galaxy.

\subsection{Metallicity distribution}

The shape of a metallicity distribution function (MDF) enables us to study possible gas inflow or outflow history in a galaxy. The MDF peak, effective yield, is a function of star formation rate, initial mass function, gas outflow rate, and lock-up mass. Analysis of MDF allows us to investigate the chemical enrichment history of a system. The MDFs of the Milky Way halo and disc have been extensively studied (Carney et al. 1996; Edvardson et al. 1993).

To obtain a MDF, stellar metallicities should be derived from the spectral data. However, spectroscopic observations for extra-Galactic systems are time-consuming even for nearby Galactic satellite dwarf galaxies by currently available telescopes. Therefore, instead of spectroscopic observations, stellar colours are frequently used to estimate metallicity distributions 

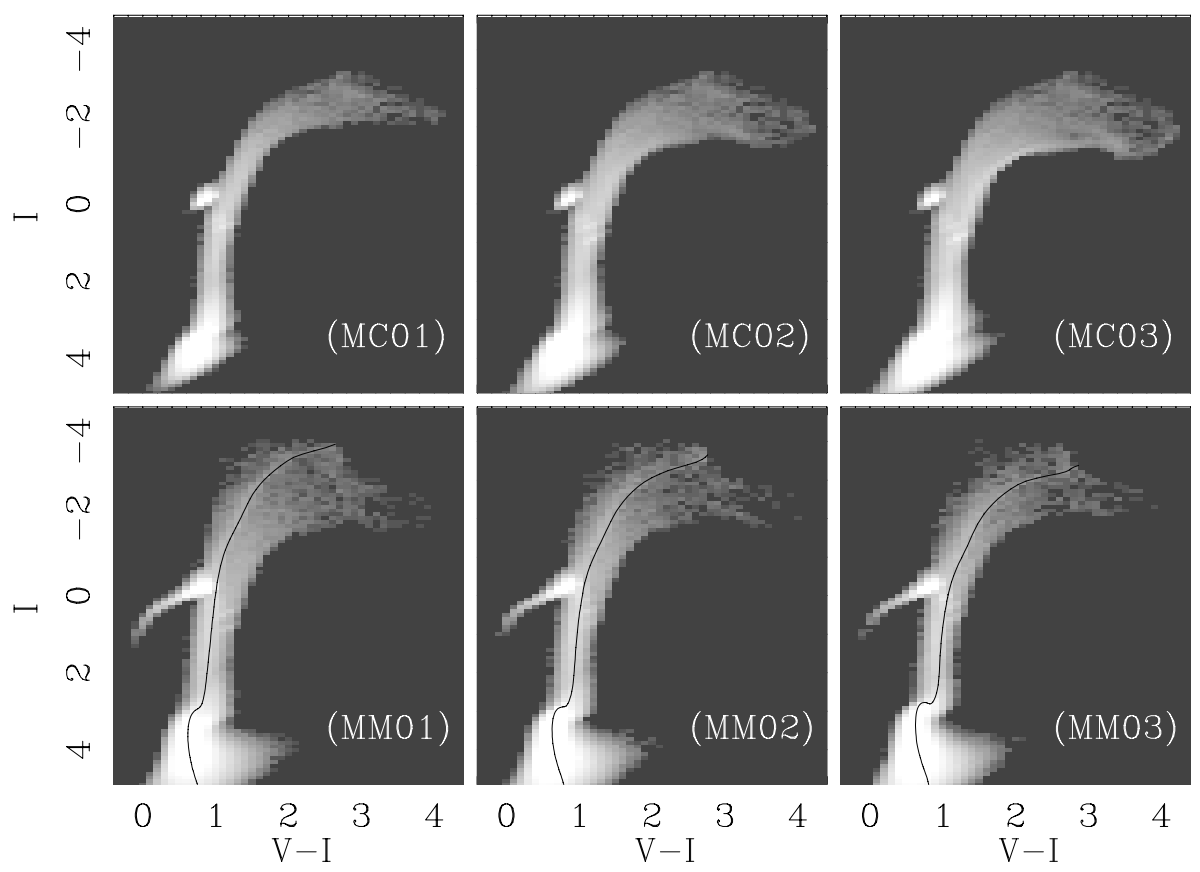

Fig. 2. Stellar density map on Hess diagrams predicted by the monolithic collapse (the upper panels) and the merging models (the lower panels). In each of the lower panels, we overplot the isochrone corresponding to the burst population.

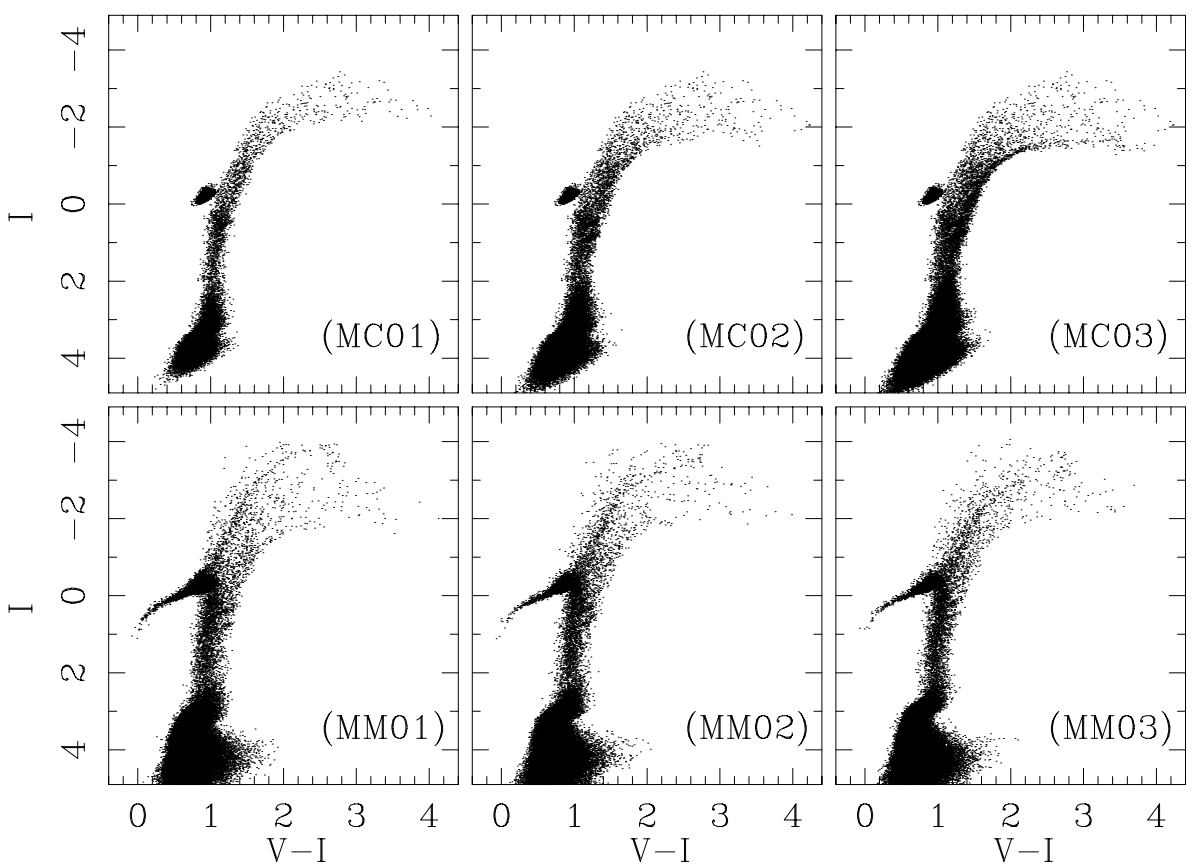

Fig. 3. C-M diagrams predicted by the monolithic collapse (the upper panels) and the merging models (the lower panels).

of remote galaxies including ellipticals (e.g., Harris \& Harris 1999, 2000, 2002).

Figures 5 and 6 show theoretical colour distributions and MDFs, respectively. In Fig. 5, the solid and dotted lines refer to the colour distribution of RGB and AGB stars of $-2.5 \leq I \leq$ -2.0 and of $-3.5 \leq I \leq-3.0$, respectively. The colour distributions obtained from the merger models have bluer peaks than those by the monolithic collapse models. Since the RGB colour mainly depends on metallicity, this reflects that higher frequency of metal-poor stars in the merger models. This tendency may help us to discriminate the two formation models.

Figure 6 shows predicted MDFs by the monolithic collapse (the upper panels) and the merger (the lower panels) models. The MDFs of the monolithic collapse models are characterised by the peaks at super-solar metallicities. On the other hand, the MDFs of the merging models show two peaks. The lower and higher metallicity peaks correspond to the yield of star formation in the progenitor galaxies and of the starburst triggered by the merging, respectively. The fractions of the burst populations relative to the total of the formed ellipticals are $0.72,0.61$, and 0.50 in the models MM01, MM02, and MM03, respectively.

The expected fractions of metal-rich stars in the two scenarios are different. Models MC01-MC03 predict a higher fraction of metal-rich stars than Models MM01-MM03. The fraction of stars of super-solar metallicity are calculated as $0.33,0.60$, and 0.76 for MC01, MC02, and MC03, respectively. Those of the merger models MM01, MM02, and MM03 are 0.28, 0.27, and 0.27 , respectively.

A comparison between the shape of the colour distribution in Fig. 5 and that of the MDF in Fig. 6 cautions that a colour distribution should be converted carefully into a MDF. For example, the lower panels in Fig. 5 show a sign of the two peaks 

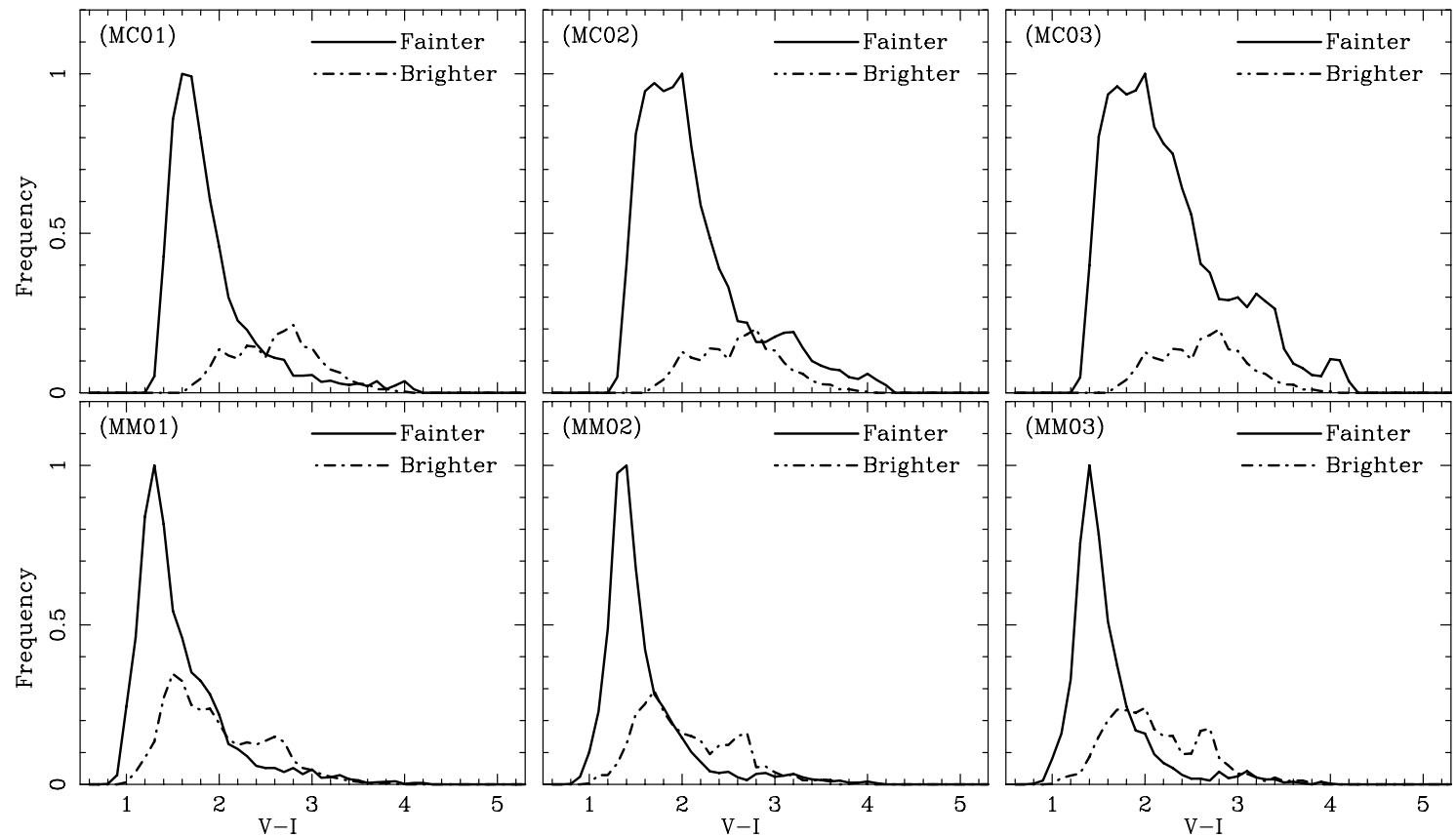

Fig. 5. Colour distribution of RGB stars predicted by the monolithic collapse (the upper panels) and the merging models (the lower panels). The solid lines show the colour distribution of RGB stars of $-2.0 \leq I \leq-1.5$, while the dotted-lines show those of $-3.0 \leq I \leq-2.5$. The colour distribution of each model is normalised at the maximum value of the distribution of fainter RGB stars.

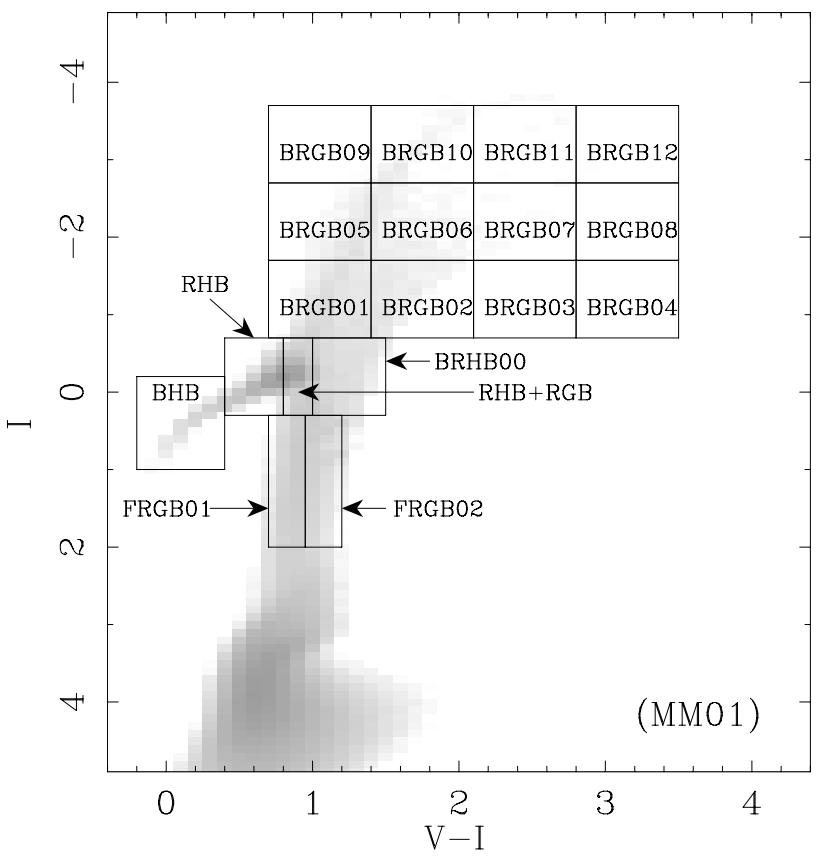

Fig. 4. Some key regions in the C-M diagrams. The fraction of stars in each box is reported in Table A.1.

of the MDFs in the colour distributions of the models MM01 and MM02, but it is not easily seen in the model MM03. It is well-known that the relation between the stellar colours and the metallicities is highly non-linear. Fractions of metal-rich stars may be underestimated if observations are not deep enough. Models MC02 and MC03 in Fig. 5 show that the colour distributions of brighter RGB and AGB stars have smaller fractions of red $(V-I>3)$ stars than those of fainter ones. Stars of higher metallicities become fainter due to line blanketing. Thus, one would miss metal-rich populations if only bright stars are taken into account to derive a MDF.

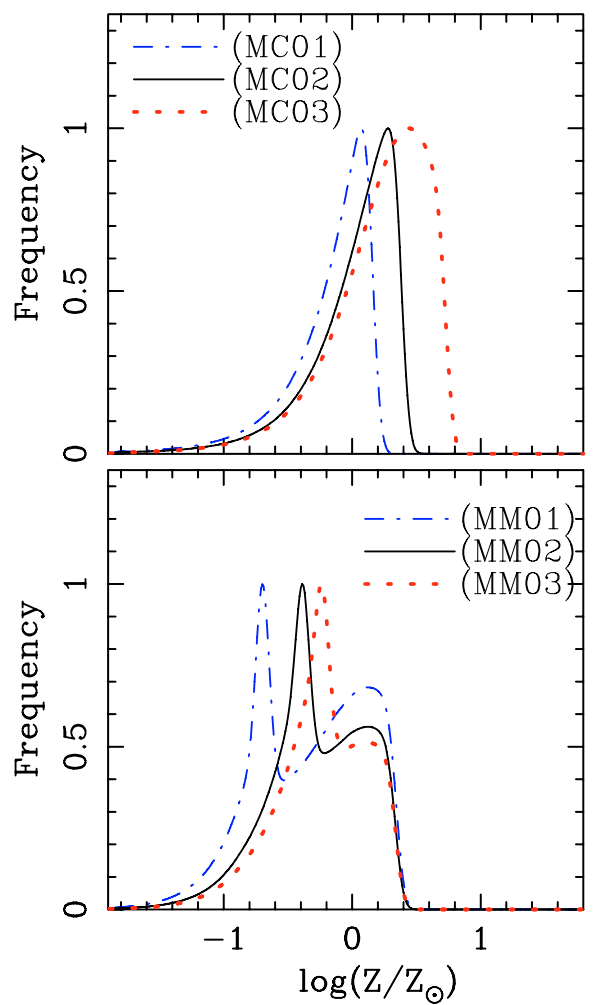

Fig. 6. MDFs predicted by the monolithic collapse (upper panel) and the merging models (lower panel). (This figure is available in color in electronic form.)

\subsection{Abundance pattern}

Figure 7 shows expected $[\mathrm{Mg} / \mathrm{Fe}]$ versus $[\mathrm{Fe} / \mathrm{H}]$, where magnesium is representative of $\alpha$ elements which are mainly produced in type II supernovae. In the monolithic collapse models, only type II supernovae drive the chemical enrichment. Thus, stars 

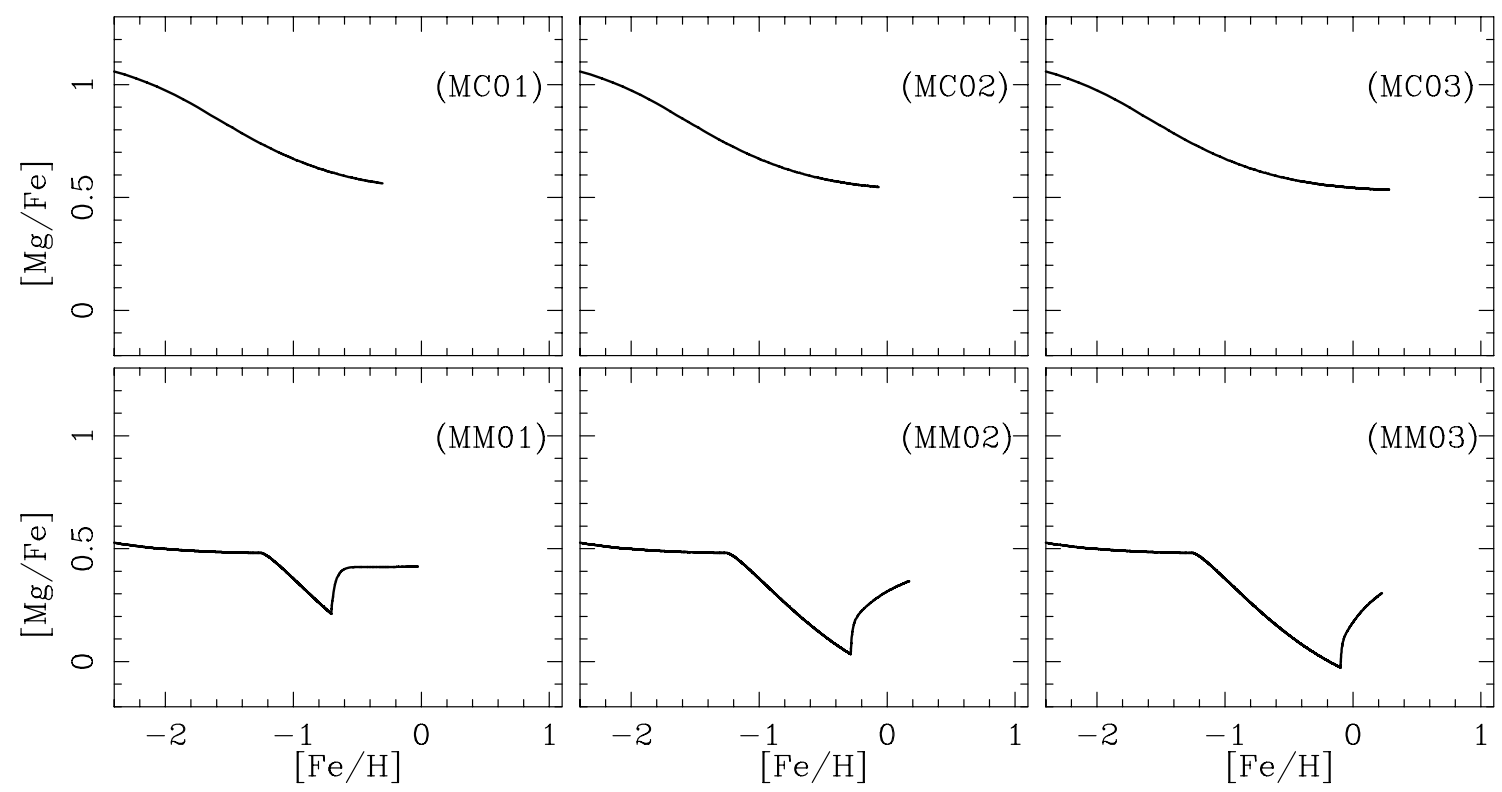

Fig. 7. Abundance ratio between an $\alpha$ element, magnesium, and iron predicted by the models. In the lower panels, i.e., prediction of the merger models, the sudden decline at $[\mathrm{Fe} / \mathrm{H}] \sim-1$ corresponds to the onset of iron enrichment by type Ia supernovae, while the next upturn results from massive $\alpha$ elements production by type II supernovae due to the star burst triggered by the merger.

have over-abundance values of $[\alpha / \mathrm{Fe}]$. On the other hand, the chemical enrichment histories of the merging models are more complicated. In the progenitor disc galaxies, type II supernovae explode and $[\alpha / \mathrm{Fe}]$ is initially over-abundant of $[\mathrm{Mg} / \mathrm{Fe}] \sim 0.5$ after 1-2 Gyr (1.5 Gyr in our chemical evolution code), type Ia supernovae begin exploding and expel plenty of iron-peak elements. This reduces $[\alpha / \mathrm{Fe}]$. When the merger triggers starburst, $[\alpha / \mathrm{Fe}]$ is pushed upward due to $\alpha$ elements newly synthesised by type II supernovae.

All models predict super-solar $[\alpha / \mathrm{Fe}]$, but the monolithic models tend to have higher values. The averaged $[\mathrm{Mg} / \mathrm{Fe}]$ of all the long-lived stars is $0.56,0.55$, and 0.54 in the models MC01, MC02, and MC03, respectively. The merger models MM01, MM02, and MM03 predict the averaged $[\mathrm{Mg} / \mathrm{Fe}]$ of $0.42,0.36$, and 0.30 , respectively.

\subsection{Integrated property}

As stated in Sect. 4.1, the morphology of the HB is the most prominent difference between the monolithic and merger models. This could appear in an integrated spectrum, in particular, in the UV region. To investigate if any differences appear in the integrated light, the spectral energy distribution (SED) of each model is calculated by means of an isochrone synthesis code. Our code to calculate SEDs gives the evolution of synthesised spectra of galaxies in a consistent manner with galaxy chemical evolution. Stellar evolutionary tracks and model atmosphere used in the code are the same as those adopted in the code to simulate a C-M diagram.

Figure 8 shows theoretical SEDs normalised at the flux at $2015 \AA$ and Table 4 gives optical colours. The SEDs of models MC03 and MM01-MM03 are similar at the optical wavelength range. In other words, the SEDs in the optical range are degenerate and it is difficult to discriminate between the scenarios. Table 4 shows the colours of each model. The colours from the $U$-band to $K$-band are similar between the models and it is difficult to discriminate the formation histories from the wavelength range.

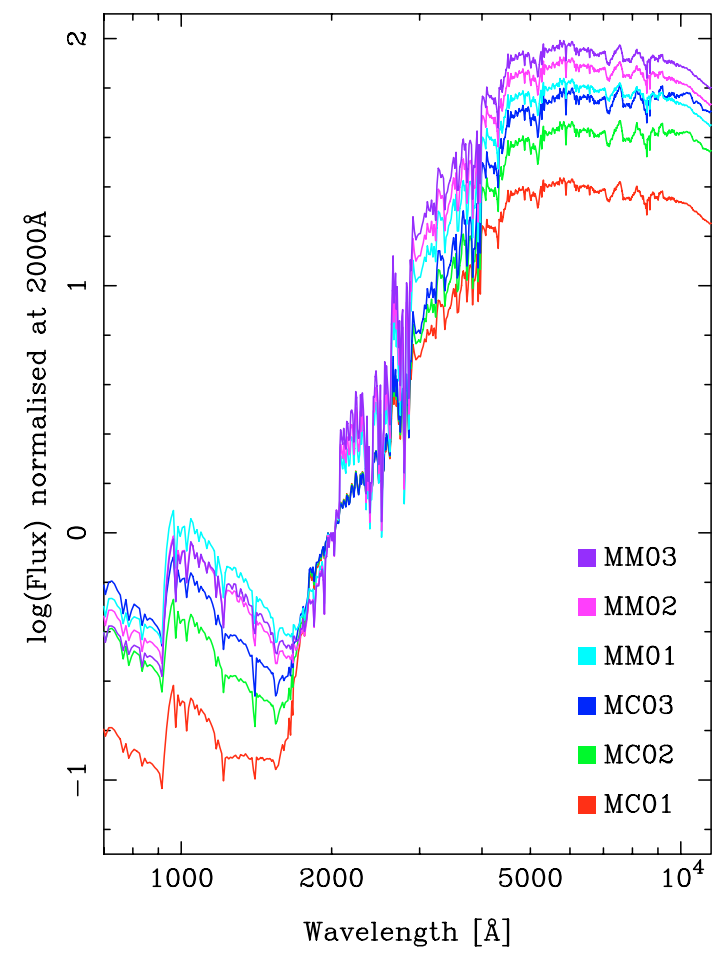

Fig. 8. SEDs of the models. The SEDs are normalised at $2015 \AA$. (This figure is available in color in electronic form.)

Table 5 summarises the UV flux. The merger models tend to have stronger UV upturn and higher flux than the monolithic ones at the wavelength range of 1000-2000 $\AA$. In particular, the flux ratio between $1095 \AA$ and $2015 \AA$ shows the largest difference between the two scenarios. The result suggests that the UV properties may help us to disentangle the competing theories of elliptical galaxy formation. However, discussing the integrated SED of the two scenarios goes beyond the scope of this paper. We do not examine here all the factors which change an 
Table 4. Optical colour calculated from the integrated SEDs.

\begin{tabular}{ccccc}
\hline \hline Model & $U-B$ & $B-V$ & $V-I$ & $V-K$ \\
\hline MC01 & 0.27 & 0.88 & 1.18 & 2.77 \\
MC02 & 0.39 & 0.95 & 1.24 & 3.19 \\
MC03 & 0.49 & 1.00 & 1.29 & 3.34 \\
MM01 & 0.35 & 0.94 & 1.23 & 2.82 \\
MM02 & 0.35 & 0.93 & 1.23 & 3.01 \\
MM03 & 0.34 & 0.92 & 1.22 & 3.02 \\
\hline
\end{tabular}

Table 5. Integrated UV flux ratios, where $F_{1045}, F_{1095}, F_{1545}$, and $F_{2015}$ mean the flux at $1045,1095,1545$, and $2015 \AA$, respectively.

\begin{tabular}{lccc}
\hline \hline Model & $\log \left(F_{1045} / F_{2015}\right)$ & $\log \left(F_{1095} / F_{2015}\right)$ & $\log \left(F_{1545} / F_{2015}\right)$ \\
\hline MC01 & -0.69 & -0.72 & -0.95 \\
MC02 & -0.35 & -0.39 & -0.76 \\
MC03 & -0.18 & -0.23 & -0.64 \\
MM01 & 0.03 & -0.02 & -0.43 \\
MM02 & -0.07 & -0.11 & -0.52 \\
MM03 & -0.07 & -0.11 & -0.48 \\
\hline
\end{tabular}

integrated SED. Further investigation is necessary if one wants to use the UV properties to discriminate the formation scenarios.

\section{Discussion and conclusion}

To illustrate what information can be obtained from C-M diagrams of elliptical galaxies, six examples of theoretical C-M diagrams are presented and discussed. These C-M diagrams can be considered as a first approach to C-M diagrams of elliptical galaxies of which formation is described as the monolithic collapse (models MC01-MC03) and the hierarchical scenario (models MM01-MM03). Differences in the C-M diagrams are; (1) the magnitude of the MSTO, (2) the HB morphology, and (3) colour distribution of RGB stars.

The magnitude of the MSTO enables us to directly measure the epoch of the most recent star formation. This strongly constrains the formation scenarios of ellipticals. However, to achieve this depth would currently be very challenging even for the nearest elliptical galaxies. For example, one of the nearest elliptical galaxy NGC 5128 is located at a distance of $3.8 \mathrm{Mpc}$ and photometry needs to reach $V \sim 32$ to estimate the epoch of the final star formation. In addition to the deep observations, one must overcome stellar crowding which hampers accurate photometry. Next generation telescopes and/or instruments are needed to investigate the formation of early-type galaxies by analysing the magnitude of the main sequence turnoff.

The second difference, the HB morphology, is a more accessible clue to the SFH of an elliptical galaxy. Observationally, the $\mathrm{HB}$ is about $3.5 \mathrm{mag}$ brighter than the main sequence turnoff and can be more easily detected. If we can obtain Hess diagrams deep enough to study the HB morphology, it would be possible to investigate the formation of elliptical galaxies. Based on our study, an elliptical galaxy of which formation and evolution are described as the hierarchical scenario, the extended blue HB should be detected in the C-M diagram. On the other hand, the C-M diagram should show few blue HB, but many red HB stars if elliptical galaxies form in an almost monolithic collapse. Although there are many uncertainties in the theory of stellar evolution at later stages such as HB, AGB etc., the HB morphology should help us to study SFH of ages in the range from a few Gyr and older. The ability to resolve the short timescale structure in the SFH necessarily worsens for older ages. Observations of the core He-burning stars such as HB and red clump stars are crucial to improve the time resolution for ages in the range from a few Gyr to the oldest ones. The distribution of stars in these structures will also provide constraints on the chemical enrichment history at intermediate and old ages. Therefore, obtaining a deep C-M diagram down to the HB level is essential to discriminate the formation scenarios of ellipticals.

Recent HST/ACS observations (Rejkuba et al. 2005) of nearby elliptical galaxy NGC 5128 show little indication of presence of blue HB stars. This agrees with the prediction by the monolithic collapse model, although Harris \& Harris (1999, $2000,2002)$ presented a more complicated halo formation scenario. By analysing the MDF of NGC 5128 derived from RGB colours, the authors discussed that the galaxy passed through two fairly distinct stages of halo formation. A very early stage starting from primordial gas in which stars are rapidly forming while the galaxy is still assembling by gas infall; and a second, more important stage of "closed-box" evolution dominated by normal star formation with little gas infall. This view can be considered as a variation of the monolithic collapse model. It is true that the elliptical galaxy NGC 5128 which has faint optical shells and plumes undoubtedly experienced accretion events. However, if the halo freezes the information of its formation era, the C-M diagram of the peculiar elliptical suggests that the monolithic collapse scenario gives a better explanation of its formation.

Thirdly, the colour distributions of the merger models have bluer peaks than those of the monolithic models. This is because the former models predict a higher fraction of metal-poor stars which formed in the progenitor galaxies with the slow chemical evolution. Comparing the colour distributions and MDFs, we discuss the dangers of deriving a MDF inappropriately by converting stellar colours.

Acknowledgements. We particularly acknowledge the referee of the paper, A. Bressan, for very important and constructive comments and suggestions. We have benefited from discussions with Chaki Kobayashi. C.I. thanks Minoru Kawamura, who provided the mental foundation stones of this work and provided important comments.

\section{References}

Arimoto, N., \& Yoshii, Y. 1987, A\&A, 173, 23

Arimoto, N., Yoshii, Y., \& Takahara, F. 1992, A\&A, 253, 21

Balluch, M. 1988, A\&A, 200, 58

Barnes J. E. 1998, in Galaxies: Interactions and Induced Star Formation, Saas-Fee Advanced Course 26. Lecture Notes 1996, Swiss Society for Astrophysics and Astronomy, XIV, ed. R. C. Kennicutt, F. Jr. Schweizer, J. E. Barnes, et al. (Berlin, Heidelberg: Springer-Verlag), 275

Brandl, B. R., \& Andersen, M. 2004, [arXiv: astro-ph/0410513]

Bertelli, G., \& Nasi, E. 2001, ApJ, 121, 1013

Bertelli, G. Bressan, A., Chiosi, C., Ng, Y. K., \& Ortolani, S. 1995, A\&A, 301, 381

Bertelli, G. Bressan, A., Chiosi, C., \& Ng, Y. K. 1996, A\&A, 310, 115

Binney, J. \& Merrifield, M. 1998, in Galactic Astronomy (Princeton: Princeton Univ. Press)

Bressan, A., Fagotto, F., Bertelli, G., \& Chiosi, C. 1993, A\&AS, 100, 647

Carney, B. W., Laird, J. B., Latham, D. W., \& Aguilar, L. A. 1996, AJ, 112, 668

Chapman, S. C., Blain, A. W., Ivison, R. J., \& Smail, I. R. 2003, Nature, 422, 695

Choi, P. I., Yan, L., Im, M., et al 2006, ApJ, 637, 241

Cole, S., Lacey, C. G., Baugh, C. M., \& Frenk, C. S. 2000, MNRAS, 319, 168

Davidge, T. J., \& van den Bergh, S. 2001, ApJ, 553, L133

Dressler, Alan, Oemler, A. Jr., Poggianti, B. M., Smail, I., et al. 2004, ApJ, 617, 867

Edvardsson, B., Andersen, J., Gustafsson, B., et al. 1993, A\&A, 275, 101

Elmegreen, B. G., \& Scalo, J. 2006, ApJ, 636, 149

Fagotto, F., Bressan, A., Bertelli, G., \& Chiosi, C. 1994a, A\&AS, 104, 365

Fagotto, F., Bressan, A., Bertelli, G., \& Chiosi, C. 1994b, A\&AS, 105, 29

Girardi, L., Bressan, A., Chiosi, C., et al. 1996, A\&AS, 117, 113

Gregg, M. D., Ferguson, H. C., Minniti, D., et al. 2004, AJ, 127, 1441 
Harris, W. E. Harris, G. L. H. \& Poole, G. B. 1999, AJ, 117, 855

Harris, W. E., \& Harris, G. L. H. 2000, AJ, 120, 2423

Harris, W. E., \& Harris, G. L. H. 2002, AJ, 123, 3108

Hernandez, X., Valls-Gabaud, D., \& Gilmore, G. 2000, MNRAS, 316, 605

Ikuta, C., \& Arimoto, N. 2000, A\&A, 358, 535

Ikuta, C., \& Arimoto, N. 2002, A\&A, 391, 55

Kauffmann, G. 1996, MNRAS, 281, 487

Kauffmann, G., White, S. D. M. \& Guiderdoni, B. 1993, MNRAS, 264, 201

Kodama, T., \& Arimoto, N. 1997, A\&A, 320, 41 (KA97)

Kuntschner, H., Ziegler, B. L., Sharples, R. M., Worthey, G., \& Fricke, K. J. 2002, A\&A, 395, 761

Larson, R. B. 1974, 4MNRAS, 166, 585

Lee, Y.-W., Demarque, P., \& Zinn, R. 1990, ApJ, 350, 155

Lejeune, Th., Cuisinier, F., \& Buser, R. 1998, A\&AS, 130, 65

Meyer-Hofmeister, E. 1969, A\&A, 2, 143

Ng, Y. K., Bertelli, G., Chiosi, C., \& Bressan, A. 1996, A\&A, 310, 771

Nomoto K., Thielemann F.-K., \& Yokoi K. 1984, ApJ, 286, 644

Rejkuba, M., Greggio, L., Harris, W. E., et al. 2005, ApJ, 631, 262
Smail, I., Ivison, E. J., Blain, Kneib, J.-P. 2002, MNRAS, 331, 495

Soria, R., Mould, J. R., Watson, A. M., et al. 1996, ApJ, 465, 79

Schulte-Ladbeck, R. E., Drozdovsky, I. O., Belfort, M., \& Hopp, U. 2003, ApSS, 284,909

Stevens, J. A., Page, M. J., Ivison, R. J., Smail, I., \& Carrera, F. J. 2004, AJ, 604, L17

Swinbank, A. M., Smail, I., \& Chapman, S. C., et al. 2004, ApJ, 617, 64

Sommer-Larsen, J., Götz, M., \& Portinari, L. 2003, ApJ, 596, 47

Takagi, T., Hanami, H., \& Arimoto, N., 2004, MNRAS, 355, 424

Thielemann, F.-K., Nomoto, K., \& Hashimoto, M. 1996, ApJ, 460, 408

Tinsley, B. M. 1972, ApJ, 178, 319

Toomre, A. 1977, in The Evolution of Galaxies and Stellar Populations, ed. B. Tinsley \& R. B. Larson (New Haven: Yale Univ. Obs.), 401

Tosi, M., Greggio, L., Marconi, G., \& Focardi, P. 1991, 1991, AJ, 102, 951

Trager, S. C., Faber, S. M., Worthey, G., \& González, J. J. 2000, AJ, 119, 1645

Walker, I. R., Mihos, J. C., \& Hernquist, L. 1996, ApJ, 460, 121

Yi, S., Demarque, P., Kim, Y.-C., et al. 2001, ApJ, 136, 417

Yan, L., Philip, I. C., Fadda, D., et al. 2004, ApJS, 154, 75 
C. Ikuta: Model colour-magnitude diagrams of elliptical galaxies $(R N)$, Online Material $p 1$

\section{Online Material}


C. Ikuta: Model colour-magnitude diagrams of elliptical galaxies $(R N)$, Online Material $p 2$

\section{Appendix A: Fraction of stars in representative \\ areas of the $\mathrm{C}-\mathrm{M}$ diagram}

Tables A.1-A.7 list the data of stars for the corresponding C-M diagram boxes shown in Fig. 4. Tables A.1 summarises stellar percentage of a given box relative to the total number of stars brighter than 2.0 in $I$-band. Tables A.2-A.7 report ratio of stars of different metallicity in a given box, where a denominator is the total number of stars in the box. 
Table A.1. Fraction of stars in each box indicated in Fig. 4 relative to the total number of stars of $I \leq 2.0$.

\begin{tabular}{crrrrrr}
\hline \hline & $\begin{array}{r}\text { MC01 } \\
(\%)\end{array}$ & $\begin{array}{r}\text { MC02 } \\
(\%)\end{array}$ & $\begin{array}{r}\text { MC03 } \\
(\%)\end{array}$ & $\begin{array}{r}\text { MM01 } \\
(\%)\end{array}$ & $\begin{array}{r}\text { MM02 } \\
(\%)\end{array}$ & $\begin{array}{r}\text { MM03 } \\
(\%)\end{array}$ \\
\hline BHB & 0.00 & 0.00 & 0.00 & 0.39 & 0.46 & 0.49 \\
RHB & 0.76 & 0.64 & 0.53 & 7.80 & 8.90 & 9.35 \\
RHB+RGB & 41.43 & 34.87 & 29.17 & 37.77 & 40.30 & 40.86 \\
BRGB00 & 17.11 & 17.61 & 17.21 & 13.28 & 12.59 & 12.55 \\
FRGB01 & 4.67 & 4.00 & 3.38 & 5.20 & 5.91 & 6.44 \\
FRGB02 & 19.67 & 20.59 & 20.17 & 14.32 & 12.87 & 12.30 \\
BRGB01 & 2.46 & 2.07 & 1.73 & 2.09 & 2.32 & 2.52 \\
BRGB02 & 5.74 & 7.46 & 8.57 & 5.73 & 4.90 & 4.51 \\
BRGB03 & 0.00 & 0.19 & 0.83 & 0.52 & 0.42 & 0.37 \\
BRGB04 & 0.00 & 0.02 & 0.20 & 0.13 & 0.10 & 0.09 \\
BRGB05 & 0.00 & 0.00 & 0.00 & 0.28 & 0.36 & 0.38 \\
BRGB06 & 3.23 & 2.82 & 2.39 & 1.88 & 1.83 & 1.88 \\
BRGB07 & 1.32 & 1.62 & 1.51 & 1.03 & 0.88 & 0.81 \\
BRGB08 & 0.42 & 0.65 & 0.66 & 0.45 & 0.39 & 0.37 \\
BRGB09 & 0.00 & 0.00 & 0.00 & 0.02 & 0.02 & 0.02 \\
BRGB10 & 0.06 & 0.05 & 0.04 & 0.21 & 0.28 & 0.32 \\
BRGB11 & 0.54 & 0.45 & 0.38 & 0.32 & 0.34 & 0.36 \\
BRGB12 & 0.48 & 0.40 & 0.34 & 0.24 & 0.21 & 0.20 \\
\hline
\end{tabular}

Table A.2. Fraction of stars of different metallicity bins in the BHB box in Fig. 4. Stars of $[\mathrm{Z} / \mathrm{H}] \geq-0.8$ do not appear in this box.

\begin{tabular}{rrrrrrr}
\hline \hline BHB & MC01 & MC02 & MC03 & MM01 & MM02 & MM03 \\
& $(\%)$ & $(\%)$ & $(\%)$ & $(\%)$ & $(\%)$ & $(\%)$ \\
\hline$[\mathrm{Z} / \mathrm{H}]<-1.0$ & 0.00 & 0.00 & 0.00 & 99.11 & 98.93 & 98.94 \\
$-1.0 \leq[\mathrm{Z} / \mathrm{H}]<-0.8$ & 0.00 & 0.00 & 0.00 & 1.01 & 1.15 & 1.13 \\
\hline
\end{tabular}

Table A.3. The same as Table A.2, but for the RHB box in Fig. 4. Stars of $[\mathrm{Z} / \mathrm{H}] \geq 0.0$ do not appear in this box.

\begin{tabular}{crrrrrr}
\hline \hline RHB & $\begin{array}{r}\text { MC01 } \\
(\%)\end{array}$ & $\begin{array}{r}\text { MC02 } \\
(\%)\end{array}$ & $\begin{array}{r}\text { MC03 } \\
(\%)\end{array}$ & $\begin{array}{r}\text { MM01 } \\
(\%)\end{array}$ & $\begin{array}{r}\text { MM02 } \\
(\%)\end{array}$ & $\begin{array}{r}\text { MM03 } \\
(\%)\end{array}$ \\
\hline$[\mathrm{Z} / H]<-1.0$ & 54.35 & 55.39 & 56.06 & 54.35 & 55.39 & 56.06 \\
$-1.0 \leq[\mathrm{Z} / \mathrm{H}]<-0.8$ & 26.46 & 26.90 & 26.92 & 26.46 & 26.90 & 26.92 \\
$-0.8 \leq[\mathrm{Z} / \mathrm{H}]<-0.6$ & 13.28 & 12.41 & 12.01 & 13.28 & 12.41 & 12.01 \\
$-0.6 \leq[\mathrm{Z} / \mathrm{H}]<-0.4$ & 2.81 & 3.00 & 3.07 & 2.81 & 3.00 & 3.07 \\
$-0.4 \leq[\mathrm{Z} / \mathrm{H}]<-0.2$ & 3.35 & 2.58 & 2.24 & 3.35 & 2.58 & 2.24 \\
$-0.2 \leq[\mathrm{Z} / \mathrm{H}]<0.0$ & 0.03 & 0.02 & 0.02 & 0.03 & 0.02 & 0.02 \\
\hline
\end{tabular}

Table A.4. The same as Table A.2, but for the RHB+RGB box in Fig. 4. Stars of $[\mathrm{Z} / \mathrm{H}] \geq 0.0$ do not appear in this box.

\begin{tabular}{crrrrrr}
\hline \hline RHB+RGB & $\begin{array}{r}\text { MC01 } \\
(\%)\end{array}$ & $\begin{array}{r}\text { MC02 } \\
(\%)\end{array}$ & $\begin{array}{r}\text { MC03 } \\
(\%)\end{array}$ & $\begin{array}{r}\text { MM01 } \\
(\%)\end{array}$ & $\begin{array}{r}\text { MM02 } \\
(\%)\end{array}$ & $\begin{array}{r}\text { MM03 } \\
(\%)\end{array}$ \\
\hline$[\mathrm{Z} / \mathrm{H}]<-1.0$ & 2.52 & 2.76 & 2.87 & 2.52 & 2.76 & 2.87 \\
$-1.0 \leq[\mathrm{Z} / \mathrm{H}]<-0.8$ & 9.15 & 10.03 & 10.46 & 9.15 & 10.03 & 10.46 \\
$-0.8 \leq[\mathrm{Z} / \mathrm{H}]<-0.6$ & 22.60 & 22.59 & 22.76 & 22.60 & 22.59 & 22.76 \\
$-0.6 \leq[\mathrm{Z} / \mathrm{H}]<-0.4$ & 20.26 & 24.43 & 26.35 & 20.26 & 24.43 & 26.35 \\
$-0.4 \leq[\mathrm{Z} / \mathrm{H}]<-0.2$ & 44.22 & 39.34 & 36.87 & 44.22 & 39.34 & 36.87 \\
$-0.2 \leq[\mathrm{Z} / \mathrm{H}]<0.0$ & 1.88 & 1.43 & 1.27 & 1.88 & 1.43 & 1.27 \\
\hline
\end{tabular}

Table A.5. The same as Table A.2, but for the BRGB00 box in Fig. 4.

\begin{tabular}{crrrrrr}
\hline \hline BRGB00 & $\begin{array}{r}\text { MC01 } \\
(\%)\end{array}$ & $\begin{array}{r}\text { MC02 } \\
(\%)\end{array}$ & $\begin{array}{r}\text { MC03 } \\
(\%)\end{array}$ & $\begin{array}{r}\text { MM01 } \\
(\%)\end{array}$ & $\begin{array}{r}\text { MM02 } \\
(\%)\end{array}$ & $\begin{array}{r}\text { MM03 } \\
(\%)\end{array}$ \\
\hline$[\mathrm{Z} / \mathrm{H}]<-1.0$ & 0.25 & 0.30 & 0.31 & 0.25 & 0.30 & 0.31 \\
$-1.0 \leq[\mathrm{Z} / \mathrm{H}]<-0.8$ & 0.95 & 1.11 & 1.19 & 0.95 & 1.11 & 1.19 \\
$-0.8 \leq[\mathrm{Z} / \mathrm{H}]<-0.6$ & 4.08 & 4.72 & 4.88 & 4.08 & 4.72 & 4.88 \\
$-0.6 \leq[\mathrm{Z} / \mathrm{H}]<-0.4$ & 4.61 & 7.54 & 8.69 & 4.61 & 7.54 & 8.69 \\
$-0.4 \leq[\mathrm{Z} / \mathrm{H}]<-0.2$ & 28.43 & 29.40 & 30.23 & 28.43 & 29.40 & 30.23 \\
$-0.2 \leq[\mathrm{Z} / \mathrm{H}]<0.0$ & 20.76 & 19.57 & 19.35 & 20.76 & 19.57 & 19.35 \\
$0.0 \leq[\mathrm{Z} / \mathrm{H}]<0.2$ & 20.00 & 18.64 & 17.98 & 20.00 & 18.64 & 17.98 \\
$0.2 \leq[\mathrm{Z} / \mathrm{H}]<0.4$ & 15.33 & 14.03 & 13.25 & 15.33 & 14.03 & 13.25 \\
$0.4 \leq[\mathrm{Z} / \mathrm{H}]<0.6$ & 4.46 & 3.81 & 3.38 & 4.46 & 3.81 & 3.38 \\
$0.6 \leq[\mathrm{Z} / \mathrm{H}]$ & 2.37 & 2.02 & 1.79 & 2.37 & 2.02 & 1.79 \\
\hline
\end{tabular}


C. Ikuta: Model colour-magnitude diagrams of elliptical galaxies $(R N)$, Online Material p 4

Table A.6. The same as Table A.2, but for FRGB which includes stars in FRGB01 and FRGB02 in Fig. 4.

\begin{tabular}{crrrrrr}
\hline \hline FRGB & $\begin{array}{r}\text { MC01 } \\
(\%)\end{array}$ & $\begin{array}{r}\text { MC02 } \\
(\%)\end{array}$ & $\begin{array}{r}\text { MC03 } \\
(\%)\end{array}$ & $\begin{array}{r}\text { MM01 } \\
(\%)\end{array}$ & $\begin{array}{r}\text { MM02 } \\
(\%)\end{array}$ & $\begin{array}{r}\text { MM03 } \\
(\%)\end{array}$ \\
\hline$[\mathrm{Z} / \mathrm{H}]<-1.0$ & 2.57 & 3.13 & 3.30 & 2.57 & 3.13 & 3.30 \\
$-1.0 \leq[\mathrm{Z} / \mathrm{H}]<-0.8$ & 2.75 & 3.34 & 3.52 & 2.75 & 3.34 & 3.52 \\
$-0.8 \leq[\mathrm{Z} / \mathrm{H}]<-0.6$ & 7.25 & 7.53 & 7.71 & 7.25 & 7.53 & 7.71 \\
$-0.6 \leq[\mathrm{Z} / \mathrm{H}]<-0.4$ & 3.04 & 5.68 & 6.82 & 3.04 & 5.68 & 6.82 \\
$-0.4 \leq[\mathrm{Z} / \mathrm{H}]<-0.2$ & 16.33 & 17.23 & 18.67 & 16.33 & 17.23 & 18.67 \\
$-0.2 \leq[\mathrm{Z} / \mathrm{H}]<0.0$ & 26.05 & 24.11 & 23.14 & 26.05 & 24.11 & 23.14 \\
$0.0 \leq[\mathrm{Z} / \mathrm{H}]<0.2$ & 25.34 & 23.41 & 22.25 & 25.34 & 23.41 & 22.25 \\
$0.0 \leq[\mathrm{Z} / \mathrm{H}]<0.2$ & 11.36 & 11.24 & 0.00 & 11.36 & 11.24 & 0.00 \\
$0.0 \leq[\mathrm{Z} / \mathrm{H}]<0.2$ & 3.48 & 2.93 & 2.59 & 3.48 & 2.93 & 2.59 \\
$0.6 \leq[\mathrm{Z} / \mathrm{H}]$ & 1.88 & 1.58 & 1.40 & 1.88 & 1.58 & 1.40 \\
\hline
\end{tabular}

Table A.7. The same as Table A.2, but for stars in BRGB which include from BRGB01 to BRGB12 boxes in Fig. 4.

\begin{tabular}{crrrrrr}
\hline \hline BRGB & $\begin{array}{r}\text { MC01 } \\
(\%)\end{array}$ & $\begin{array}{r}\text { MC02 } \\
(\%)\end{array}$ & $\begin{array}{r}\text { MC03 } \\
(\%)\end{array}$ & $\begin{array}{r}\text { MM01 } \\
(\%)\end{array}$ & $\begin{array}{r}\text { MM02 } \\
(\%)\end{array}$ & $\begin{array}{r}\text { MM03 } \\
(\%)\end{array}$ \\
\hline$[\mathrm{Z} / \mathrm{H}]<-1.0$ & 1.91 & 2.40 & 2.56 & 1.91 & 2.40 & 2.56 \\
$-1.0 \leq[\mathrm{Z} / \mathrm{H}]<-0.8$ & 2.01 & 2.53 & 2.73 & 2.01 & 2.53 & 2.73 \\
$-0.8 \leq[\mathrm{Z} / \mathrm{H}]<-0.6$ & 4.90 & 5.74 & 5.95 & 4.90 & 5.74 & 5.95 \\
$-0.6 \leq[\mathrm{Z} / \mathrm{H}]<-0.4$ & 2.46 & 4.77 & 5.83 & 2.46 & 4.77 & 5.83 \\
$-0.4 \leq[\mathrm{Z} / \mathrm{H}]<-0.2$ & 14.23 & 15.57 & 17.05 & 14.23 & 15.57 & 17.05 \\
$-0.2 \leq[\mathrm{Z} / \mathrm{H}]<0.0$ & 21.74 & 20.67 & 20.26 & 21.74 & 20.67 & 20.26 \\
$0.0 \leq[\mathrm{Z} / \mathrm{H}]<0.2$ & 24.31 & 22.72 & 21.80 & 24.31 & 22.72 & 21.80 \\
$0.2 \leq[\mathrm{Z} / \mathrm{H}]<0.4$ & 18.59 & 17.15 & 16.25 & 18.59 & 17.15 & 16.25 \\
$0.4 \leq[\mathrm{Z} / \mathrm{H}]<0.6$ & 7.16 & 6.20 & 5.57 & 7.16 & 6.20 & 5.57 \\
$0.6 \leq[\mathrm{Z} / \mathrm{H}]$ & 3.83 & 3.32 & 2.98 & 3.83 & 3.32 & 2.98 \\
\hline
\end{tabular}

